\title{
Hybrid anisotropic plasmonic metasurfaces with multiple resonances of focused light beams
}

Yao Liang ${ }^{1,2, \S}$, Han Lin $^{2, \S}$, Shirong Lin ${ }^{3}$, Jiayang Wu ${ }^{4}$, Weibai Li $^{2}$, Fei Meng ${ }^{2}$, Yunyi Yang ${ }^{2}$, Xiaodong Huang ${ }^{2}$, Baohua Jia ${ }^{2, *}$ and Yuri Kivshar ${ }^{1, *}$

${ }^{1}$ Nonlinear Physics Center, Research School of Physics, Australian National University, Canberra, ACT 2601, Australia

${ }^{2}$ Centre of Translational Atomaterials (CTAM), Faculty of Science, Engineering and Technology, Swinburne University of Technology, Hawthorn, VIC 3122, Australia

${ }^{3}$ State Key Laboratory of Advanced Optical Communication Systems and Networks, School of Physics and Astronomy, Shanghai Jiao Tong University, Shanghai 200240, China

${ }^{4}$ Optical Sciences Centre, Swinburne University of Technology, Hawthorn, VIC 3122, Australia §These authors contribute equally

*Corresponding authors: bjia@swin.edu.au (Baohua Jia); ysk@internode.on.net (Yuri Kivshar) 


\section{Methods}

Sample fabrication. Our sample is fabricated in two steps. First, the anisotropic arrays ( $42 \times 42$ units) are fabricated using two-photon polymerization techniques by a femtosecond laser (Libra, $100 \mathrm{fs}, 10 \mathrm{kHz}, 800 \mathrm{~nm}$ ) and a silicon-zirconium hybrid photoresist (negative tone) ${ }^{1}$. Next, we metalize the laser fabricated sample by uniformly coating 100-nm-thick gold on it using multiple physical vapor depositions ${ }^{2}$.

FTIR Measurements. Reflection spectra are measured using an FTIR system (Bruker Vertex 70) connected to a microscope (Bruker Hyperion 2000), for which the schematic is shown in Figure S4a in Supporting Information. Before measurements, the mercury cadmium telluride (MCT) detector is cooled by liquid nitrogen. To reduce the portion of TE-polarized light coming from undesired directions ${ }^{3}$, we partially block the reflective objectives (Figure S4c, Supporting Information), since TE polarized light acts as background light, which does not excite any resonance of interest (Figure S1, Supporting Information). We used the unpatterned gold film as the background reference and choose a spectral resolution of $4 \mathrm{~cm}^{-1}$. To improve the signal-to-noise ratio, we averaged 400 scans for all measurements. Reflection signals are taken from a small area of $\sim 25 \times 25 \mu \mathrm{m}^{2}$ by using an aperture.

Numerical Modeling. We simulated the reflection spectra and the far-field intensities employing a commercial software of FDTD solutions (Lumerical). For the tips of pillars and walls, semi-ellipse cross-sections are used to better represent the laser fabricated sample. We use a uniform mesh size of $20 \mathrm{~nm}$ (x/y-and z-directions) within a unit cell except at the vicinities of the tips of the pillar and the grating walls, where 3D non-uniform meshes are applied with a minimum size of $5 \mathrm{~nm}$. Perfect matching layer (PML) conditions are applied along the z-direction while periodic boundary conditions in the $x$ - and $y$-directions. For oblique incidences, we use the Broadband Fixed Angle Source Technique (BFAST) option in the plane wave source. For far-field diffraction calculation, a $10 \times 10$ finite array size is assumed to better represent the experimental measurement setup. The refractive index ( $n$ ) of $\mathrm{SiO}_{2}$ and photoresist are set as 1.46 for all wavelengths. The refractive index ( $n$ ) of gold for all simulation is taken from the data of Palik ${ }^{4}$, except the one in Figure S7 of Supporting Information, where a Drude model is used, $\varepsilon(\omega)=1-\omega_{p}^{2} /\left(\omega^{2}+i \gamma \omega\right)$, where the plasma frequency $\omega_{p}=1.55 \times 10^{16} \mathrm{~s}^{-1}$ and the damping parameter $\gamma=9.2 \times 10^{13} \mathrm{~s}^{-1}$ for the $100 \mathrm{~nm}$ golden film ${ }^{5,6}$. 


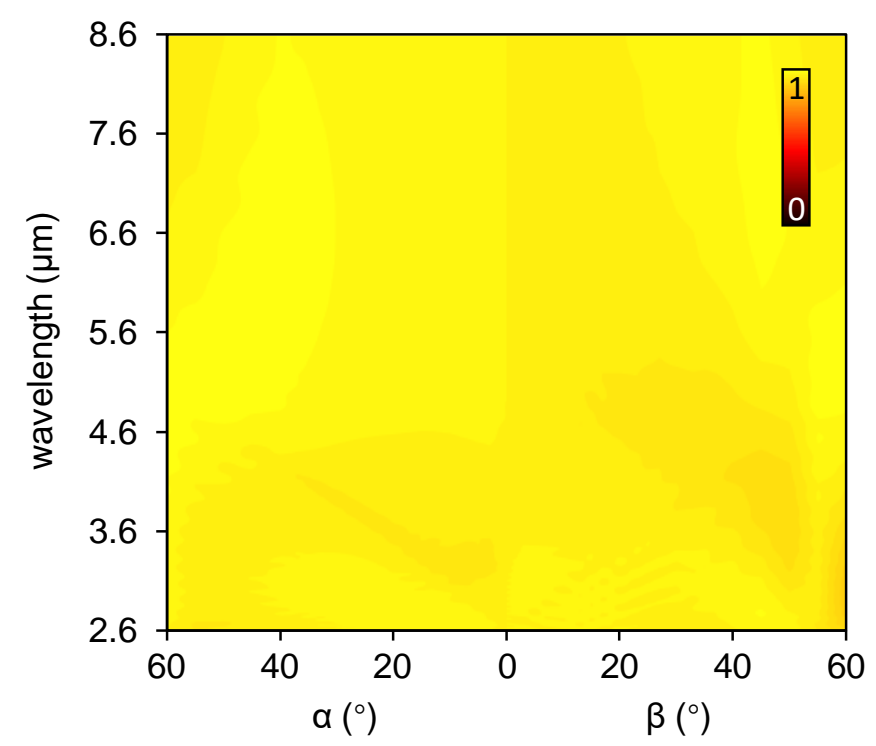

Figure S1. TE band diagram. FDTD simulated angle-resolved reflection spectra (band diagrams) under transverse electric (TE) polarized plane waves illuminations in xz-plane $(\beta)$ and yz-plane $(\alpha)$, respectively. The TE polarization in oblique incidence is defined as light with an E-field vector is orthogonal to the TM polarization (see Figure 1a in the main text). 

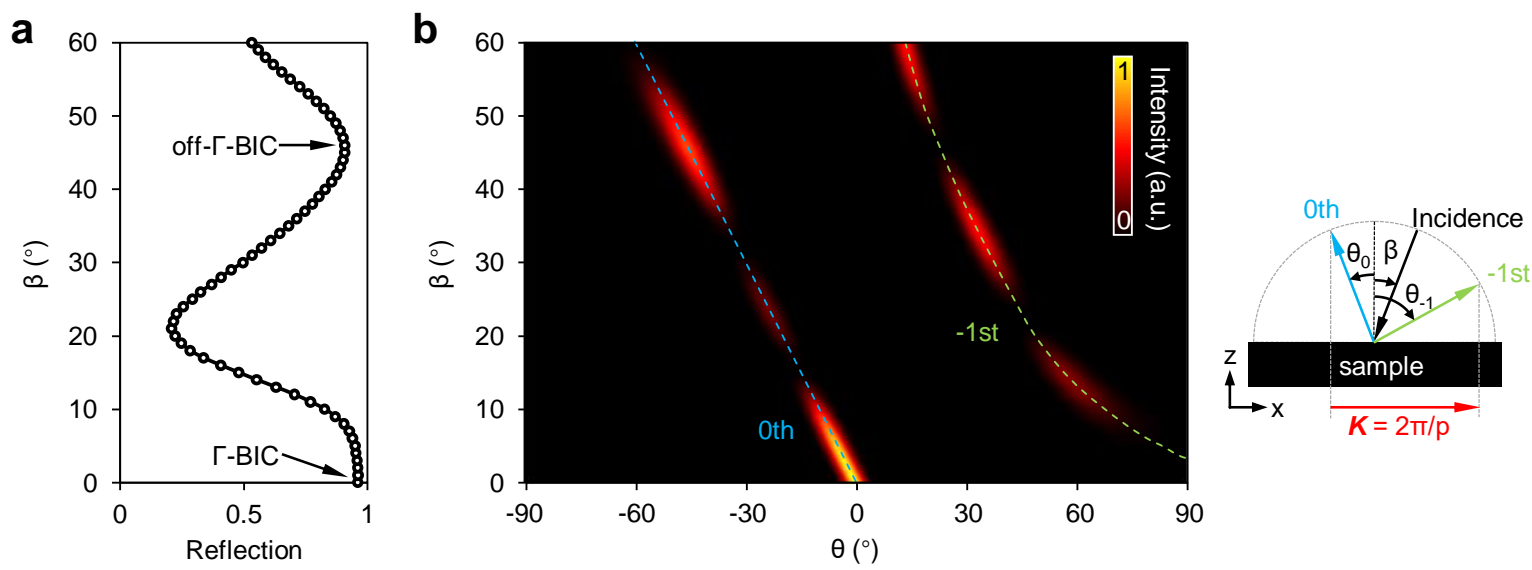

Figure S2. Resonance induced far-field diffractions. (a) FDTD simulated reflection for the resonance wavelengths of "Mode 1" under TM polarized light illumination for various oblique incidence angles $\beta$. Data are taken from Figure $2 \mathrm{a}$ in the main text. The arrows indicate the positions of $\Gamma$-BIC and off- $Г-B I C$. (b) Left: Simulated 2D contour for far-field reflection intensity $\left(|\mathrm{E}|^{2}\right)$ as a function of diffractive angles $\theta$ (x-axis) and incidence angle $\beta$ (y-axis) for a finite size metasurface (10×10 units). The light blue and light green dotted lines show the center position of the 0th and -1 st diffractions. Right: The relationship between the 0th and -1 st diffracted light, where $\beta=\theta_{0}$ and $\frac{2 \pi}{\lambda} \sin \left(\theta_{0}\right)-K=\frac{2 \pi}{\lambda} \sin \left(-\theta_{-1}\right)$ and $K=\frac{2 \pi}{P}$ is the reciprocal lattice vector. 
a

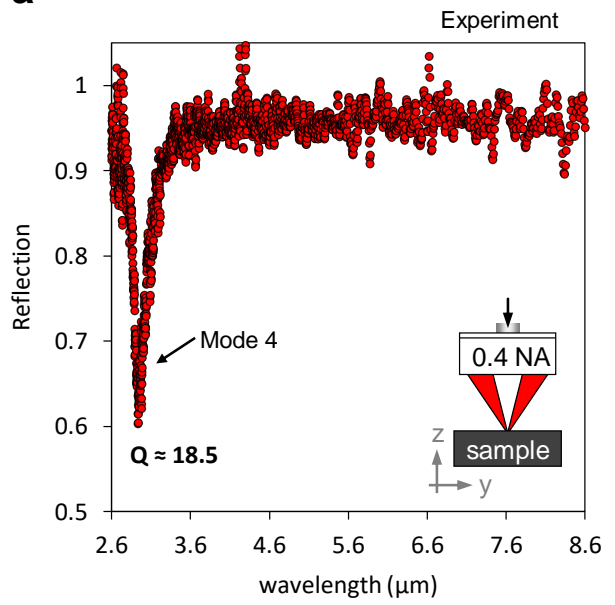

b

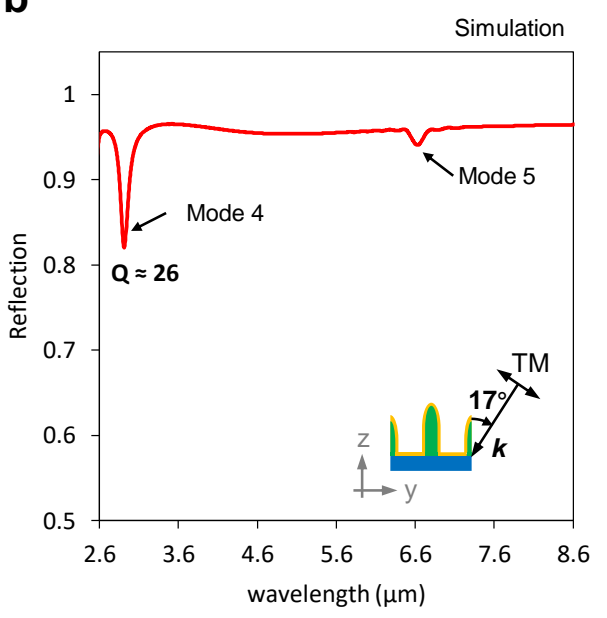
Mode $4 @ \lambda=2.91 \mu \mathrm{m}$

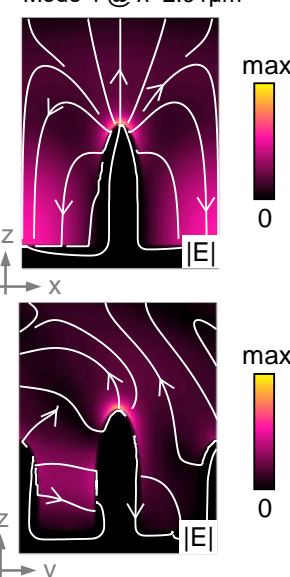

Figure S3. "Mode 4" measurement. (a) FTIR measured spectrum for TM polarization in the yz-plane using a partial blocked reflective objective ( $N A=0.4$, see Figure $S 4$ for details). (b) Left: the FDTD simulated reflection spectrum for TM polarized light illumination in the yz-plane (oblique angle $\alpha=17^{\circ}$ ). Data are taken from Figure $2 a$ in the main text. Right: the electric field distributions of "Mode 4" at the resonance wavelength $\lambda=2.91 \mu \mathrm{m}$ in the $x z-$ and $y z$-planes. The white streamlines show the instantaneous field directions. 
a

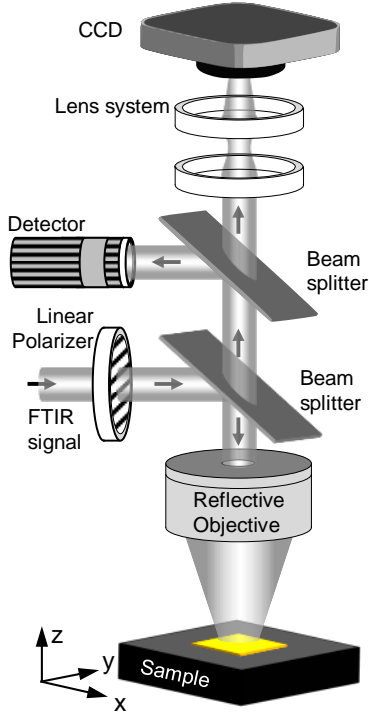

b

\begin{tabular}{|c|c|c|c|}
\hline NA & $\mathrm{D}(\mathrm{mm})$ & $\eta 1\left(^{\circ}\right)$ & $\mathrm{\eta}\left(^{\circ}\right)$ \\
\hline 0.4 & 24 & 12 & 23.6 \\
\hline 0.5 & 10.4 & 15 & 30 \\
\hline 0.65 & 7.5 & 19.4 & 40.5 \\
\hline
\end{tabular}

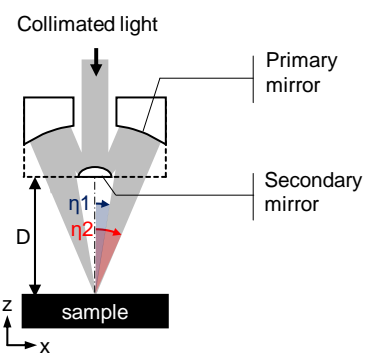

C
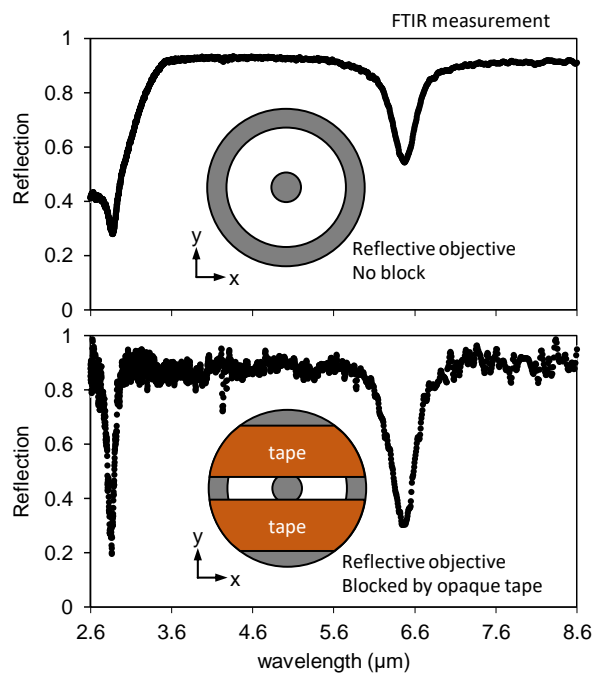

Figure S4. Measurement system. (a) Schematics of the FTIR measurement system. CCD stands for charge coupled device. (b) A cross-section view of a reflective objective without chromatic aberrations, where light is focused on the sample through a hollow light cone between inner angle ( $\eta 1)$ and outer angle ( $\eta 2)$. The inner/outer angles and working distance (D) for different numerical aperture (NA) objectives are different, as shown in the top panel table. (c) Reflection spectra measured by the FTIR system using an unblock-objective and a partial-blocked-objective (NA $=0.4)$. 

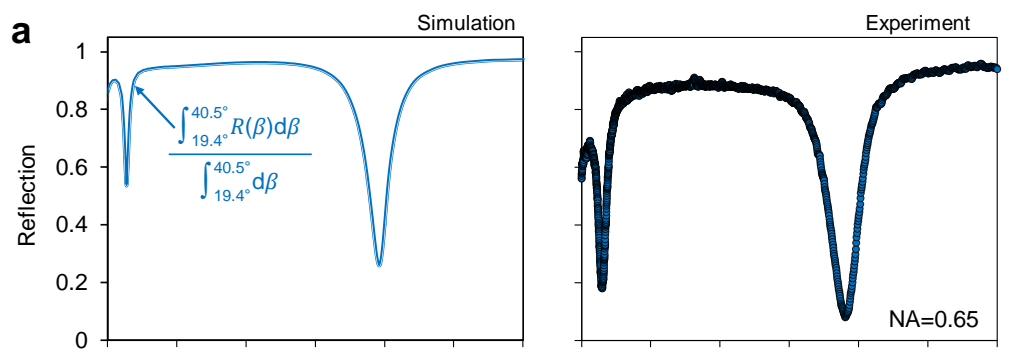

b
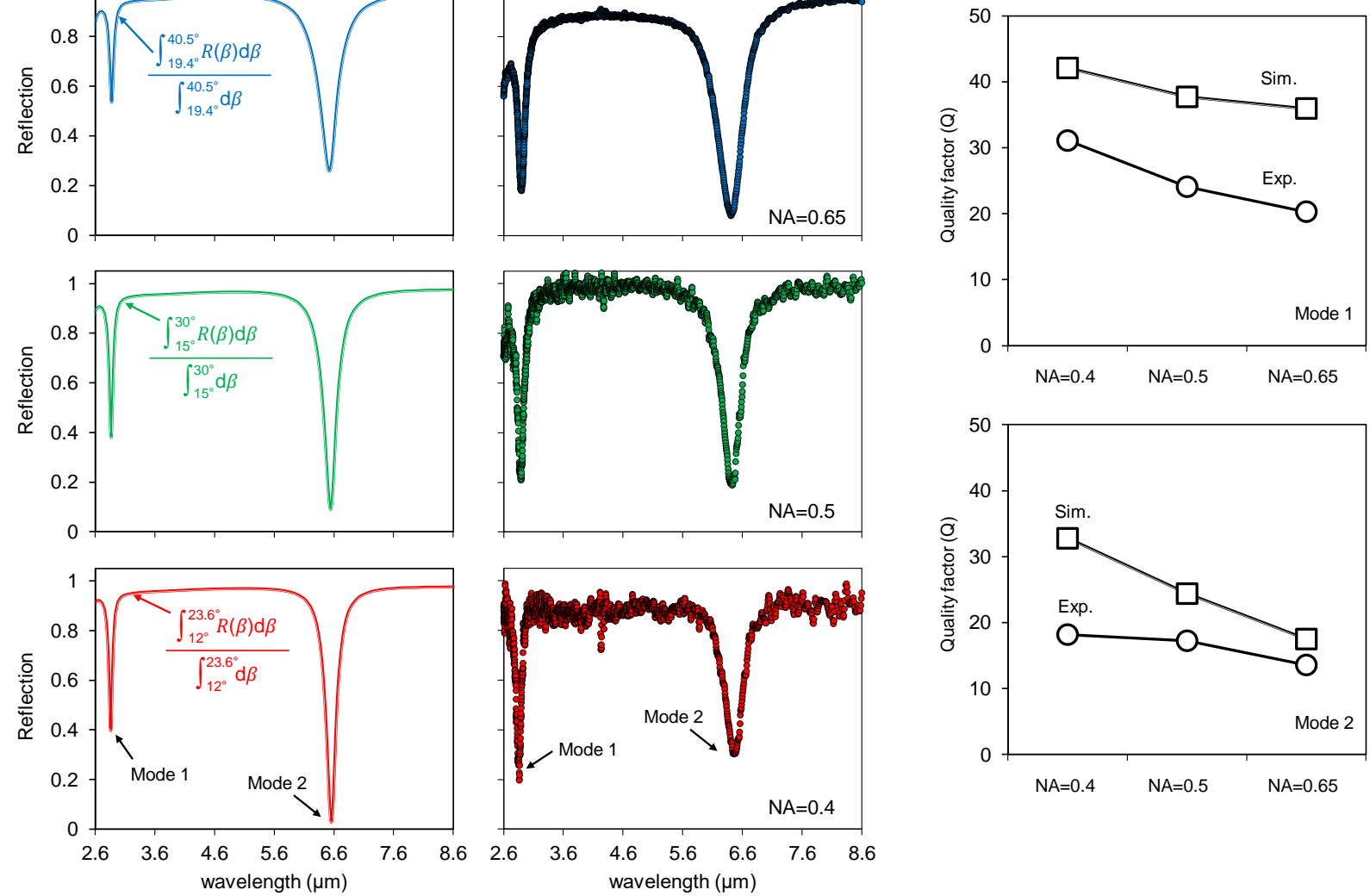

Figure S5. Comparison of experiment and theory. (a) Left: The FDTD simulation average results of multipole oblique angle incidence in the xz-plane $\left(\frac{\int_{\eta 1}^{\eta 2} R(\beta) \mathrm{d} \beta}{\int_{\eta 1}^{\eta 2} d \beta}\right.$, where $R(\beta)$ is the reflection spectrum at oblique angle $\beta$ that is taken from Figure $2 \mathrm{a}$ in the main). The angles $\eta 1,2$ are chosen based on the hollow light cone angles for different NA reflective objectives (see Figure S4b for details). Right: the FTIR measurement results using partial blocked reflective objectives with various numerical apertures (NA $=0.4,0.5,0.65)$. (b) The dependence of resonances quality $(Q)$ factors on various numerical apertures for "Mode 1" and "Mode 2". The $\mathrm{Q}$ factors are extracted from Figure S5a using $Q=\lambda / \Delta \lambda$. Simulation results and experimental results are respectively shown by square and circular dots. 

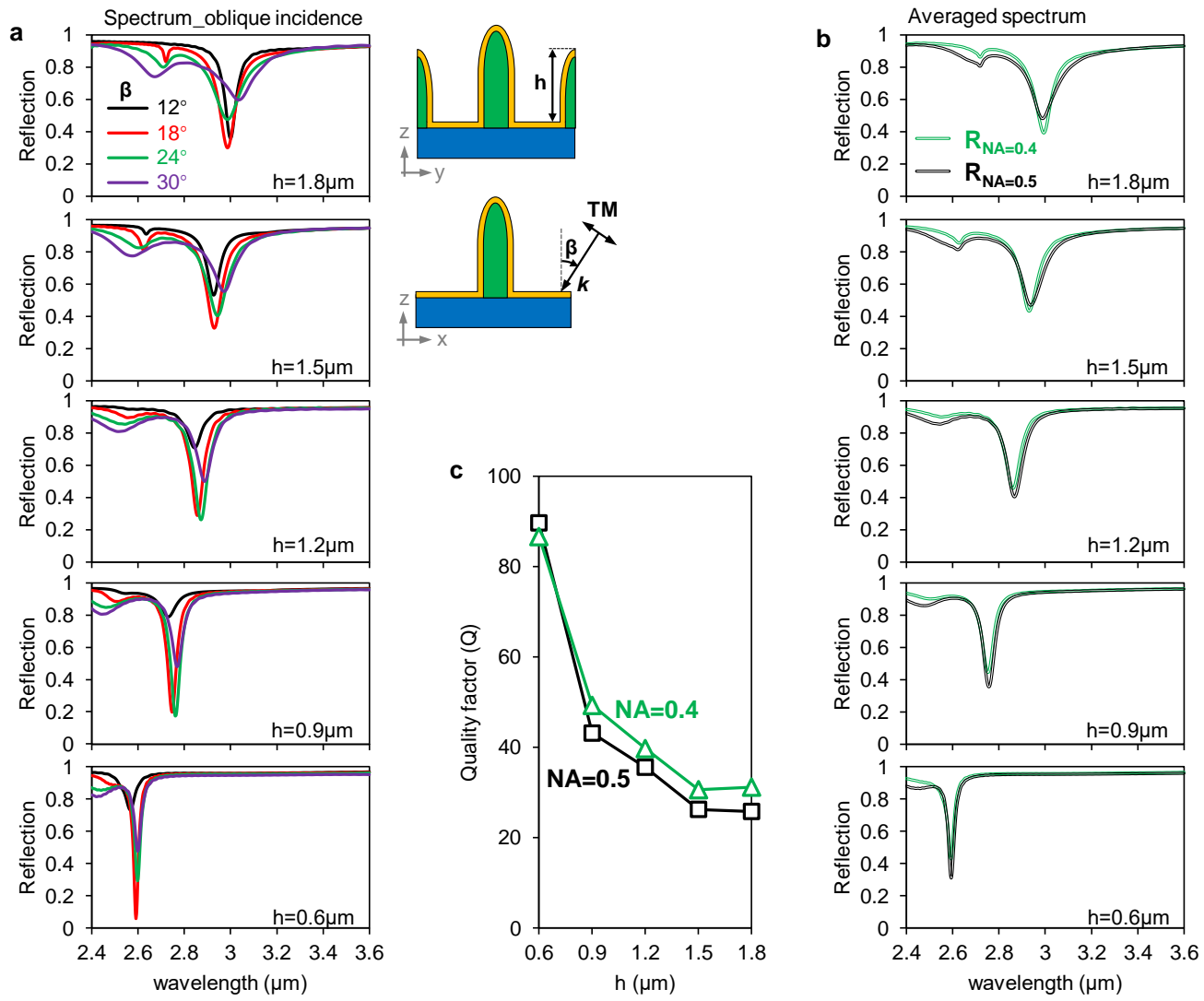

Figure S6. Geometric parameters discussion. (a) Reflection spectra for our metasurfaces with different geometric parameters $h$ (the height of the grating wall) at various oblique incidence angles $\beta$ in the xz-plane under TM-polarized light illumination. (b) The estimated spectra average results of metasurfaces (different parameter $h$ ) for two reflective objectives, $N A=0.4$ and $N A=0.5$, which respectively, collect light from angle spans $12^{\circ}-23.6^{\circ}$ and $15^{\circ}-30^{\circ}$ (see Figure S4b for details). The averaged spectra for NA $=0.4$ and NA $=0.5$ reflective objectives are, respectively, roughly estimated using $R_{N A=0.4}=\left[R\left(12^{\circ}\right)+R\left(18^{\circ}\right)+R\left(24^{\circ}\right)\right] / 3$ and $R_{N A=0.5}=\left[R\left(18^{\circ}\right)+R\left(24^{\circ}\right)+R\left(30^{\circ}\right)\right] / 3$, where $R(\beta)$ is the reflection spectrum of oblique incidence (at oblique angle $\beta$ ), which is taken from Figure S6a. (c) The estimated $Q$-factor dependence on the geometric parameter $\mathrm{h}$ for two objectives. Data extracted from Figure S6b. 
a
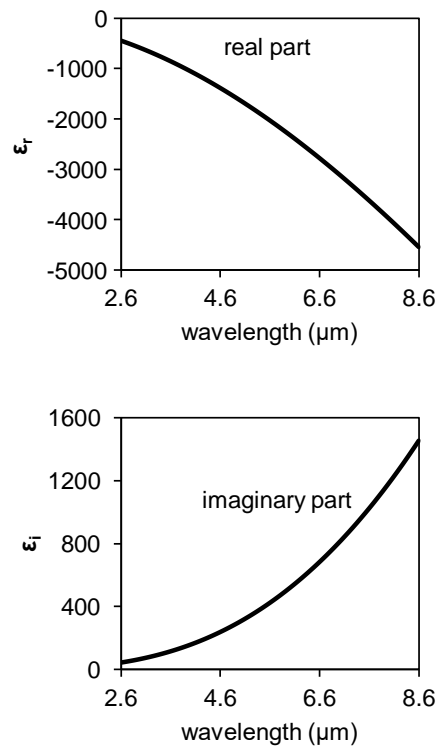

b

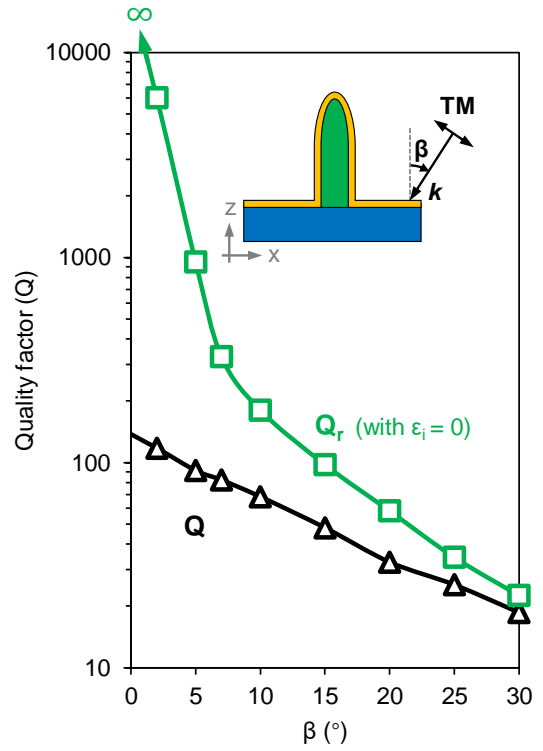

Figure S7. Q-factor discussion of "Mode 2". (a) The real $\left(\varepsilon_{r}\right)$ and imaginary $\left(\varepsilon_{i}\right)$ parts of the permittivity of gold, which is calculated using a Drude model $\varepsilon(\omega)=1-\omega_{p}^{2} /\left(\omega^{2}+i \gamma \omega\right)$, where the plasma frequency $\omega_{p}=$ $1.55 \times 10^{16} \mathrm{~s}^{-1}$ and the damping parameter $\gamma=9.2 \times 10^{13} \mathrm{~s}^{-1}$ for the $100 \mathrm{~nm}$ golden film ${ }^{5,6}$. (b) FDTD simulated quasi-BIC $Q$ factors (total $Q$-factor $(Q)$, and the radiative $Q$-factor, $Q_{r}$ ) versus the incidence angle $\beta$ in the xz-plane for "Mode 2" (see Figure 2a in the main text). The total Q-factors are extracted from the linewidths of resonant spectra simulations using the material data (gold) shown in Figure S7a. For the radiative Q-factors $\left(Q_{r}\right)$, they are extracted from simulations with "lossless" gold, where the imaginary part of the permittivity of gold is set to zero $\left(\varepsilon_{i}(\omega)=0\right)$. Correspondingly, the $\mathrm{Q}$-factor is determined sorely by $\mathrm{Q}^{-1}=Q_{r}^{-1}$ since $Q_{d}=\infty$. 

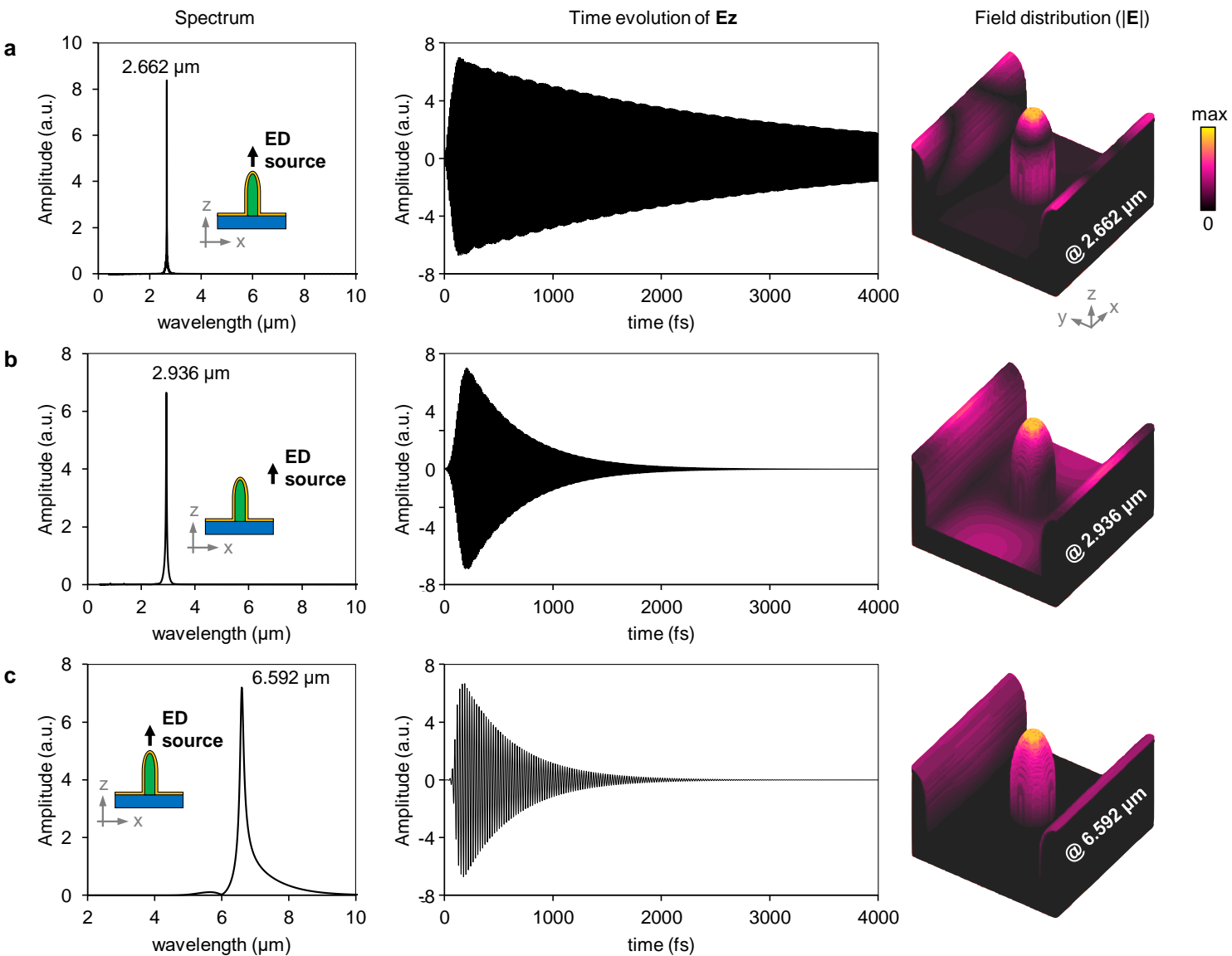

Figure S8. Near-field excitation of BICs. A vertical dynamic electric dipole (ED) source (subradiant) in the near field is used to excite various BICs modes due to the large overlap between them. A point monitor is placed on the top of the middle pillar. The corresponding spectrum and E-field evolution (Ez dephasing) and the E-field distribution $(|\mathrm{E}|)$ are shown. To excite BICs resonance at different wavelengths, the position of ED source and its center wavelength and pulse length are changed correspondingly. (a) ED source on the top of the middle pillar with a center wavelength at $2.6 \mu \mathrm{m}$ and the pulse length of $80 \mathrm{fs}$ (b) ED source in the middle of two adjacent pillars with a center wavelength at $2.9 \mu \mathrm{m}$ and the pulse length of $80 \mathrm{fs}$. (c) ED source on the top of the middle pillar with a center wavelength at $6.6 \mu \mathrm{m}$ and the pulse length of $39 \mathrm{fs}$. 


\section{References}

1. Terzaki K, Vasilantonakis N, Gaidukeviciute A, et al. 3D conducting nanostructures fabricated using direct laser writing. Optical Materials Express. 2011;1(4):586-597.

2. Liang Y, Lin H, Koshelev K, et al. Full-stokes polarization perfect absorption with diatomic metasurfaces. Nano Letters. 2021;21(2):1090-1095.

3. Liang $\mathrm{Y}$, Koshelev $\mathrm{K}$, Zhang $\mathrm{F}$, et al. Bound states in the continuum in anisotropic plasmonic metasurfaces. Nano Letters. 2020;20(9):6351-6356.

4. Palik, E. D. Handbook of Optical Constants of Solids; Academic Press: 1998.

5. Yakubovsky DI, Arsenin AV, Stebunov YV, Fedyanin DY, Volkov VS. Optical constants and structural properties of thin gold films. Optics express. 2017;25(21):25574-25587.

6. Stebunov YV, Yakubovsky DI, Leiman V, Arsenin AV, Volkov VS. Influence of the crystalline structure of metal films on the performance of plasmonic biosensors. Paper presented at: 3rd International Conference on Metamaterials and Nanophotonics, METANANO 20182018. 